\title{
Bimodality treatment of patients with pelvic adenoid cystic carcinoma with photon intensity- modulated radiotherapy plus carbon ion boost:
} a case series

This article was published in the following Dove Press journal: Cancer Management and Research

\author{
Denise Bernhardt ${ }^{1-3}$ \\ Florian Sterzing ${ }^{1-4,5}$ \\ Sebastian Adeberg ${ }^{1-4}$ \\ Klaus Herfarth ${ }^{1-3}$ \\ Sonja Katayama ${ }^{1,3}$ \\ Robert Foerster ${ }^{1,3,6}$ \\ Juliane Hoerner-Rieber ${ }^{1,3}$ \\ Laila König',3 \\ Juergen Debus ${ }^{1-4}$ \\ Stefan Rieken ${ }^{1,3}$ \\ 'Department of Radiation Oncology, \\ University Hospital of Heidelberg, \\ Heidelberg, Germany; ${ }^{2}$ Heidelberg \\ Ion-Beam Therapy Center (HIT), \\ Heidelberg, Germany; ${ }^{3}$ Heidelberg \\ Institute of Radiation Oncology \\ (HIRO), Heidelberg, Germany; \\ ${ }^{4}$ Clinical Cooperation Unit Radiation \\ Oncology, German Cancer Research \\ Center (DKFZ), Heidelberg, Germany; \\ ${ }^{5}$ Department of Radiation Oncology, \\ Klinikum Kempten, Kempten, \\ Germany; ${ }^{6}$ Department of Radiation \\ Oncology, University Hospital of \\ Zürich, Zürich, Switzerland
}

\begin{abstract}
Background: Treatment of patients with pelvic adenoid cystic carcinoma (ACC) remains a challenge owing to the rarity of the disease, the lack of data, and the relative radioresistance of these tumors.

Case reports: This case series presents the results of three patients with recurrent or inoperable pelvic ACC treated with intensity-modulated radiotherapy (IMRT) plus carbon ion (C12) boost. Patients received $\mathrm{C} 12$ therapy at a dose of 3 Gray equivalents (GyE) (relative biological effectiveness [RBE]) per fraction up to $24 \mathrm{GyE}$ RBE, followed by $50 \mathrm{GyE}$ of photon IMRT in 25 fractions. Conclusion: IMRT plus $\mathrm{C} 12$ ion boost as a definitive or adjuvant treatment for pelvic ACCs seems to be a promising therapeutic option. No unexpected toxicity was detected and the observed toxicity remained consistently low. The initial treatment response is promising and similar to that experienced for head and neck ACCs.
\end{abstract}

Keywords: ACC of Bartholin's gland, adenoid cystic carcinoma, ACC of the prostate, ion therapy, IMRT, tomotherapy, particle irradiation, carbon ion

\section{Introduction}

Given the rarity of pelvic adenoid cystic carcinomas (ACCs), there have been no prospective trials to determine the optimal therapy for these tumors. ACCs are rare and comparatively chemoresistant and radioresistant tumors, located mainly in the head and neck area. They are the predominant histological type among malignancies of the minor salivary glands and the submandibular gland. ${ }^{1}$ Besides the head and neck area, they can arise from sites not associated with salivary tissues, such as breast, ${ }^{2}$ vulva, ${ }^{3}$ cervix, ${ }^{4}$ and prostate. ${ }^{5}$ The World Health Organization (WHO) classification has combined ACC of the prostate with other malignant basaloid tumors of the prostate under the single term basal cell carcinoma (BCC). ${ }^{6}$ Accordingly, there are only a few case reports of prostatic ACCs, with the largest series of 19 cases reported by Iczkowski et al. $^{7}$ Most of these patients received radical surgery as first-line management, such as transurethral resection of the prostate, radical prostatectomy, or pelvic exenteratio.

In female patients, ACC of Bartholin's gland is a rare malignancy of the vulva. So far, treatment with a wide local excision, with or without ipsilateral inguinal lymphadenectomy and with or without adjuvant radiotherapy, ${ }^{3,8-11}$ has been recommended as the optimal treatment. Adjuvant radiotherapy lowers the local recurrence in patients with positive resection margins. ${ }^{8}$ Furthermore, ACCs of Bartholin's gland are likely to extend deeply into the lateral wall of the vagina and infiltrate the ischiorectal fossa.
Correspondence: Denise Bernhardt Department of Radiation Oncology, University Hospital of Heidelberg, Im Neuenheimer Feld 400, D-69120 Heidelberg, Germany

Tel +49622I 5635654

Fax +49622156565353

Email denise.bernhardt@med.uniheidelberg.de 
Therefore, it may be difficult to remove the entire lesion, and patients with inoperable or gross residual disease remain a particular challenge. ${ }^{12}$

Independent of their origin, ACCs show aggressive local behavior with extensive infiltration, and they are characterized by multiple local relapses and a tendency for delayed recurrence and metastasis after initial treatment. ${ }^{8,13,14}$ It is postulated that the standard treatment for ACCs is radical surgical resection, ensuring free margins, ${ }^{13,15}$ but the extensive local infiltrative and perineural spread often causes difficulty in achieving high tumor control. Prognostic factors such as advanced tumors, positive resection margins, and perineural infiltration have been an indication for postoperative radiotherapy, although most data are available only for head and neck ACCs. ${ }^{15-18}$ In the metastatic situation, case series of head and neck ACCs suggest that the median survival is about 3 years, but some patients survive longer than 10 years. ${ }^{19,20}$ Therefore, the potential toxicity of palliative treatment should be weighed against the fact that many patients have slowly progressive disease that can cause few symptoms for several years. Especially in the setting of inoperable, incompletely resected, or recurrent disease, treatment remains a therapeutic challenge. In advanced stages, conventional chemotherapy regimens are still used; however, few of these agents have shown efficacy. ${ }^{21,22}$ To date, the optimal treatment strategy for ACC outside the head and neck area remains unclear. Most data are limited to case reports owing to the rarity of the disease, which is further allocated to different medical specialties. Mutilating surgical procedures such as pelvic exenteration are often performed as a last option for these patients, since, so far, there is no valid treatment alternative.

Jensen et al showed that photon intensity-modulated radiotherapy (IMRT) plus a carbon ion (C12) boost for locally advanced inoperable or subtotally resected ACC in the head and neck resulted in significantly improved local control as well as overall survival compared to photon IMRT alone..$^{23,24}$ There was no significant difference between patients with inoperable and partially resected ACC.

\section{Case reports}

We report three cases of pelvic ACCs treated in accordance with the treatment given for head and neck ACCs, with IMRT plus $\mathrm{C} 12$ heavy ion boost, in a single institution between April 2014 and March 2016.

\section{Ethics}

Written informed consent was obtained from all patients for publication of this case report and any accompanying images.

\section{Treatment}

According to the experiences with head and neck ACCs, we applied radiotherapy as photon IMRT plus C12 heavy ion boost as an individual treatment concept. We administered radiotherapy within a bimodal concept, consisting of a first phase of carbon ion irradiation (boost) to areas of macroscopic residues or with an increased risk of recurrence, and a second phase of photon IMRT. The C12 treatment was performed at the Heidelberg Ion-Beam Therapy Center (Heidelberg, Germany) using the intensity-controlled rasterscanned technique (active beam application, with one or two lateral beams). The first clinical target volume (CTV1) for the carbon ion boost included the macroscopic tumor and/or tumor bed. The first planning target volume (PTV1) consisted of a $3 \mathrm{~mm}$ margin around CTV1. Patients received 24 Gray equivalents (GyE) (relative biological effectiveness [RBE]) in $3 \mathrm{GyE}$ (RBE) per fraction (five or six fractions per week). Doses were prescribed to the median PTV, which should be encompassed by the $95 \%$ prescription isodose. Organs at risk were contoured and adapted on the basis of computed tomography (CT) scans and magnetic resonance imaging (MRI). Dose constraints of normal tissue were respected according to QUANTEC reports. ${ }^{25,26}$

After the up-front C12 treatment, we performed photon IMRT of the pelvic lymphatic drainage. Patient 3 also received irradiation of the inguinal lymph drainage. CTV2 included the macroscopic tumor or tumor bed and the lymph drainage. PTV 2 consisted of a $3 \mathrm{~mm}$ margin around CTV2. IMRT was performed using a TomoTherapy ${ }^{\circledR}$ accelerator with $6 \mathrm{MV}$ photon beam up to $50 \mathrm{GyE}$ using helical tomotherapy (Accuray, Sunnyvale, CA, USA) in 25 fractions (five fractions per week) (Figure 1).

All patients were seen by a physician several times during radiation therapy and at regular follow-up intervals. MRI of the abdomen and pelvis was recommended every $2-3$ months after irradiation. CT scans of the chest were recommended every 12 months. Treatment-related toxicity was documented according to the most up-to-date version of the Common Terminology Criteria for Adverse Events (CTCAE) v4.0 classification. ${ }^{27}$

\section{Case I \\ Case presentation}

We present the case of a 65-year-old Caucasian male (Karnofsky performance status [KPS] 80\%) who was initially diagnosed with prostate cancer in March 2012 at another institution. Prostate-specific antigen (PSA) levels were elevated and biopsy of the prostate showed an adenocarcinoma 

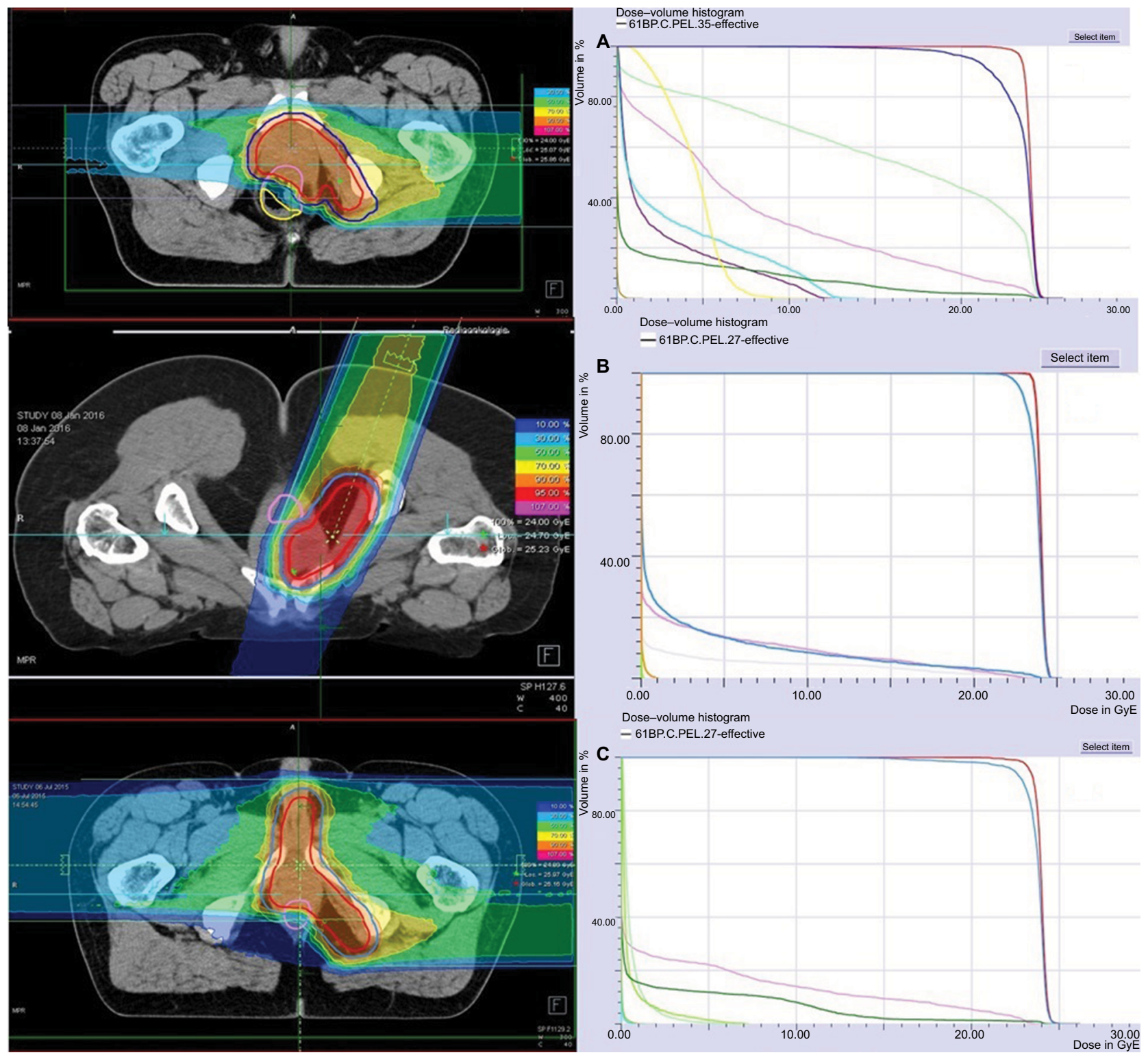

Figure I Dose distribution and dose-volume histogram of boost plans with a carbon ion total dose of 24 GyE (RBE); isodoses in percent. (A) Case I; (B) case 2; (C) case 3. Abbreviations: RBE, relative biological effectiveness; GyE, Gray equivalents.

with a Gleason score of 6 . A CT scan of the pelvis revealed a synchronous small tumor of the perirectal soft tissue infiltrating the rectum and the pelvic floor. The patient underwent radical prostatectomy and a biopsy of the second tumor site was taken during this surgery. The prostatic cancer was diagnosed as a pT2c, pN0, L0, V0, R0 adenocarcinoma of the prostate. Pathological examination of the second tumor reportedly revealed histological characteristics of a tumor with "basal cell carcinoma patterns." No further treatment was initiated until, in February 2014, a CT scan showed a local progression of the pelvic tumor. The patient underwent rebiopsy and pathological reexamination of the first biopsy. Based on these findings, a diagnosis of ACC of the pelvic floor was established. Histologically, the tumor did not originate from the prostate and was not infiltrating the prostate.

The patient presented at our institution in March 2014. 2-Fluoro-2-deoxy-D-glucose positron emission tomography/ CT showed accumulations in the pelvic floor and perirectal soft tissue as a sign of a locally advanced and aggressive tumor. Furthermore, a solitary bone metastasis was diagnosed in the left ischium. Surgical options were limited and the only reasonable approach seemed to be pelvic exenteration, which the patient refused (Figure 2A). Therefore, definitive IMRT plus C12 heavy ion boost was applied as an individual treatment.

Treatment was tolerated with moderate acute and late toxicity. During radiotherapy, the patient had mild diarrhea 



Figure 2 (A) Pretreatment MRI (TI_tra), case I; (B) follow-up MRI (TI_tra) 6 weeks after treatment, case I; (C) pretreatment MRI (TI_dixon_tra), case 2; (D) follow-up MRI (TI_tra) 5 months after treatment, case 2 . Arrows indicate primary tumor.

Abbreviation: MRI, magnetic resonance imaging.

(common toxicity criteria [CTC] grade 1) and acute urinary retention requiring catheterization. The patient already had urinary incontinence (CTC grade 2-3) before radiotherapy, and this did not worsen during radiotherapy. At the end of treatment, the skin in the radiation field showed an erythema (CTC grade 1). Furthermore, he had mild pain in the ischium, which was located in the irradiation field.

\section{Outcome and follow-up}

The patient still had urinary incontinence and mild pain in the ischium. Stools and defecation were normal after radiotherapy (Figure 2B). In September 2015, 16 months after treatment, MRI scans revealed a progression of the initial tumor region in the pelvic floor with an infiltration of the rectum but no further signs of pathological lymph nodes. PSA levels were not elevated. After multidisciplinary consultation and with the patient's consent, pelvic exenteration was performed. A pathological evaluation established a diagnosis of $\mathrm{BCC}$ with ACC-like patterns. The carcinoma showed p63-positive basaloid cells with high expression of BCL2 and EGFR. There was no expression of chromogranin A, synaptophysin, or alpha-methylacyl-CoA racemase, or translocation of $6 q 23$. Slightly positive immunostaining for p63 and expression of androgen receptors were found in about $80 \%$ of the cells.
Ki67 staining was $10 \%$. A CT scan of the chest taken at this time showed multiple small pulmonary nodes.

In February 2016, an MRI scan of the pelvis showed a residual and stable bone metastasis in the ischium but no further signs of tumor progression or recurrence. A follow-up CT scan of the chest revealed a slowly growing pulmonary metastasis. Chemotherapy with gemcitabine and carboplatin was initiated at another hospital.

\section{Case 2}

\section{Case presentation}

We further present the case of a 44-year-old Caucasian male (KPS 90\%) with an ACC of the pelvic floor and the perineum, who was first seen in our department in July 2015.

The patient had been well until 2 years before this presentation, when he noted perirectal pain. In May 2015, the patient presented at another university hospital for evaluation after the pain level increased. In the clinical examination, a rigidification was found in the perineal area. PSA levels were within the normal range. Pathological examination disclosed an ACC. CT and MRI of the chest, abdomen, and pelvis revealed a tumor of the perineum which was infiltrating the penile cavernous body and the prostate (Figure 2C). No pathological pelvic lymph nodes were found. Similarly 
to our first case, treatment options were limited to surgery and would be mutilating for the patient. After multidisciplinary consultation, we performed bimodal radiotherapy as described in the "Treatment" section.

During radiotherapy from July to September 2015, the patient had dysuria (CTC grade 2), pollakiuria (CTC grade 1), and worsening of the perineal swelling, with perirectal pain and pain during defecation. At the end of treatment, he had an erythema (CTC grade 2) in the radiation field with no signs of desquamation. Virility, stools, and defecation were normal.

\section{Outcome and follow-up}

Six weeks after radiotherapy, all symptoms including the perirectal pain had decreased. The patient still had mild pollakiuria. Virility, stools, and defecation were normal after radiotherapy, while the KPS was $80 \%$. MRI scans showed a partial remission with residual contrast enhancement in the pelvic floor (Figure 2D), the cavernous body, and the prostate, and no signs of progression. We continued the follow-up every 3 months. At the latest follow-up, 24 months after ion therapy, there were no new pathological findings.

\section{Case 3}

\section{Case presentation}

A 50-year-old female (KPS 90\%) presented at our institution in January 2015 with a diagnosis of a recurrent ACC of Bartholin's gland. Initially, the patient had been diagnosed in 2011 at another institution after presenting with a painless, gradually increasing vulvar mass. Hemivulvectomy with unilateral superficial and deep inguinal lymph-node dissection was performed and revealed a pT1, pN0 (0/14), R0, L0, V0, M0 ACC of Bartholin's gland. Four years later, in August 2015, she presented with a $5 \mathrm{~cm}$ diameter recurrence in the same region but now also infiltrating the pelvic floor. A second local excision was performed but the resection was judged to be macroscopically incomplete, which was confirmed by the pathological findings. Postoperative MRI scans showed a residual tumor infiltrating the pelvic floor, the lateral vaginal wall, and the levator ani muscle. No suspect lymph nodes or distant metastases were found.

During radiotherapy from January 2016 to March 2016, the patient had dysuria and pollakiuria. At the end of treatment, the skin in the radiation field showed a mild erythema (CTC grade 2) with moist vaginal and inguinal desquamation. The patient had diarrhea (CTC grade 1) with no need for medication.

\section{Outcome and follow-up}

Six weeks after radiotherapy, the patient was in good condition (KPS 80\%) and all symptoms including the moist desquamation had decreased. She still had tolerable vaginal pain. Stools and defecation have been normal since radiotherapy. The patient was provided with a vaginal dilator kit and instructed to use the dilator three or four times per week to avoid vaginal stenosis. MRI scans showed a partial remission with a residual contrast enhancement in the pelvic floor and no signs of progression. We continued the follow-up every 3 months. The latest follow-up, 16 months after ion therapy, did not reveal any new pathological findings.

\section{Conclusion}

For all of the presented patients, the alternative to bimodality treatment would have been a mutilating pelvic exenteration, potentially not very efficient chemotherapy, or best supportive care. All patients were relatively young and had a good performance status. Therefore, the preservation of quality of life was extremely important. Notably, patients in this report tolerated therapy well with the use of bimodality treatment and dose delivery up to $24 \mathrm{GyE} \mathrm{C} 12$ plus 50 GyE photons. No unexpected toxicity was detected and the observed toxicity remained consistently low. The most significant adverse effect was an acute urinary retention and an erythema (CTC grade 2) with moist desquamation.

Considering the extensive and mutilating surgical procedures used, definitive, adjuvant, or postoperative radiotherapy using our new approach offers a treatment alternative to patients with pelvic ACC, especially for those with locally advanced disease. Owing to the multiple local recurrences seen in ACCs, some authors postulate that radiation probably delays rather than prevents recurrences. However, from our point of view, delaying a morbid surgical procedure is to be considered as a positive treatment outcome in terms of quality of life. The initial treatment response is promising and similar to our experience for head and neck ACCs, although longer follow-up is needed to assess local control. ACCs of the pelvis are likely to recur locally by extensive infiltration of the pelvic floor, the skeletal pelvis, and the lateral pelvic wall, which should be considered in treatment planning and target definition. Because of the limited number of patients treated, additional data and longer follow-up will be required to validate the efficacy of this approach. However, our initial findings suggest that the use of C12 therapy followed by photon IMRT may be considered for patients with locally advanced pelvic ACC.

\section{Acknowledgments}

This work was supported by the Medical Faculty of Heidelberg University providing a research grant for JHR, LK, and 
DB. The data set supporting the conclusions of this article is available on request from the corresponding author.

\section{Disclosure}

The authors report no conflicts of interest in this work.

\section{References}

1. Seifert G, Brocheriou C, Cardesa A, Eveson JW. WHO international histological classification of tumours. Tentative histological classification of salivary gland tumours. Pathol Res Pract. 1990;186(5):555-581.

2. Boujelbene N, Khabir A, Jeanneret Sozzi W, Mirimanoff RO, Khanfir K. Clinical review - breast adenoid cystic carcinoma. Breast. 2012;21(2):124-127.

3. DePasquale SE, McGuinness TB, Mangan CE, Husson M, Woodland MB. Adenoid cystic carcinoma of Bartholin's gland: a review of the literature and report of a patient. Gynecol Oncol. 1996;61(1):122-125.

4. Young RH, Clement PB. Endocervical adenocarcinoma and its variants: their morphology and differential diagnosis. Histopathology. 2002;41(3): 185-207.

5. Humphrey PA. Histological variants of prostatic carcinoma and their significance. Histopathology. 2012;60(1):59-74.

6. McKenney JK, Amin MB, Srigley JR, et al. Basal cell proliferations of the prostate other than usual basal cell hyperplasia: a clinicopathologic study of 23 cases, including four carcinomas, with a proposed classification. Am J Surg Pathol. 2004;28(10):1289-1298.

7. Iczkowski KA, Ferguson KL, Grier DD, et al. Adenoid cystic/basal cell carcinoma of the prostate: clinicopathologic findings in 19 cases. $\mathrm{Am} \mathrm{J}$ Surg Pathol. 2003;27(12):1523-1529.

8. Yang S-YV, Lee J-W, Kim W-S, et al. Adenoid cystic carcinoma of the Bartholin's gland: report of two cases and review of the literature. Gynecol Oncol. 2006;100(2):422-425.

9. Morita Y, Hikage S, Ogino M. Adenoid cystic carcinoma of Bartholin's gland. Int J Gynecol Obstet. 1996;54(3):279-280.

10. Cardosi RJ, Speights A, Fiorica JV, Grendys EC, Hakam A, Hoffman MS. Bartholin's gland carcinoma: a 15-year experience. Gynecol Oncol. 2001;82(2):247-251.

11. Anaf V, Buxant F, Rodesch F, et al. Adenoid cystic carcinoma of Bartholin's gland: what is the optimal approach? Eur J Surg Oncol. 1999;25(4):406-409.

12. Rosenberg P, Simonsen E, Risberg B. Adenoid cystic carcinoma of Bartholin's gland: a report of five new cases treated with surgery and radiotherapy. Gynecol Oncol. 1989;34(2):145-147.

13. Sung M-W, Kim KH, Kim J-W, et al. Clinicopathologic predictors and impact of distant metastasis from adenoid cystic carcinoma of the head and neck. Arch Otolaryngol Head Neck Surg. 2003;129(11):1193-1197.

14. Chen AM, Bucci MK, Weinberg V, et al. Adenoid cystic carcinoma of the head and neck treated by surgery with or without postoperative radiation therapy: prognostic features of recurrence. Int J Radiat Oncol Biol Phys. 2006;66(1):152-159.
15. Garden AS, Weber RS, Morrison WH, Ang KK, Peters LJ. The influence of positive margins and nerve invasion in adenoid cystic carcinoma of the head and neck treated with surgery and radiation. Int J Radiat Oncol Biol Phys. 1995;32(3):619-626.

16. Silverman DA, Carlson TP, Khuntia D, Bergstrom RT, Saxton J, Esclamado RM. Role for postoperative radiation therapy in adenoid cystic carcinoma of the head and neck. Laryngoscope. 2004;114(7): 1194-1199.

17. Mendenhall WM, Morris CG, Amdur RJ, Werning JW, Hinerman $\mathrm{RW}$, Villaret DB. Radiotherapy alone or combined with surgery for adenoid cystic carcinoma of the head and neck. Head Neck. 2004;26(2): 154-162.

18. Chen AM, Granchi PJ, Garcia J, Bucci MK, Fu KK, Eisele DW. Localregional recurrence after surgery without postoperative irradiation for carcinomas of the major salivary glands: implications for adjuvant therapy. Int J Radiat Oncol Biol Phys. 2007;67(4):982-987.

19. Spiro RH. Distant metastasis in adenoid cystic carcinoma of salivary origin. Am J Surg. 1997;174(5):495-498.

20. Spiro RH, Huvos AG. Stage means more than grade in adenoid cystic carcinoma. Am J Surg. 1992;164(6):623-628.

21. Papaspyrou G, Hoch S, Rinaldo A, et al. Chemotherapy and targeted therapy in adenoid cystic carcinoma of the head and neck: a review. Head Neck. 2011;33(6):905-911.

22. Laurie SA, Ho AL, Fury MG, Sherman E, Pfister DG. Systemic therapy in the management of metastatic or locally recurrent adenoid cystic carcinoma of the salivary glands: a systematic review. Lancet Oncol. 2011;12(8):815-824.

23. Jensen AD, Nikoghosyan AV, Poulakis M, et al. Combined intensitymodulated radiotherapy plus raster-scanned carbon ion boost for advanced adenoid cystic carcinoma of the head and neck results in superior locoregional control and overall survival. Cancer. 2015;121(17): 3001-3009.

24. Jensen AD, Poulakis M, Nikoghosyan AV, et al. High-LET radiotherapy for adenoid cystic carcinoma of the head and neck: 15 years' experience with raster-scanned carbon ion therapy. Radiother Oncol. 2016;118(2):272-280.

25. Bentzen SM, Constine LS, Deasy JO, et al. Quantitative Analyses of Normal Tissue Effects in the Clinic (QUANTEC): an introduction to the scientific issues. Int J Radiat Oncol Biol Phys. 2010;76(3 Suppl): S3-S9.

26. Marks LB, Yorke ED, Jackson A, et al. Use of normal tissue complication probability models in the clinic. Int J Radiat Oncol Biol Phys. 2010;76(3 Suppl):S10-S19.

27. NIH National Cancer Institute. Common Terminology Criteria for Adverse Events v4.0. 2009; Bethesda, MD: National Cancer Institute. Available from: https://evs.nci.nih.gov/ftp1/CTCAE/ CTCAE_4.03_2010-06-14_QuickReference_5x7.pdf. Accessed January 30,2018 .
Cancer Management and Research

\section{Publish your work in this journal}

Cancer Management and Research is an international, peer-reviewed open access journal focusing on cancer research and the optimal use of preventative and integrated treatment interventions to achieve improved outcomes, enhanced survival and quality of life for the cancer patient. The manuscript management system is completely online and includes

\section{Dovepress}

a very quick and fair peer-review system, which is all easy to use. Visit http://www.dovepress.com/testimonials.php to read real quotes from published authors. 